\title{
Buffer Analysis Parallel Optimized Algorithm of Vector Data Based on Plane Graph Model
}

\author{
Zhanlong Chen ${ }^{\mathrm{a}}$, Liang $\mathrm{Wu}^{\mathrm{b}, *}$ \\ Faculty of Information Engineering, China University of Geosciences, Wuhan \\ 430074, China \\ ${ }^{a}$ chenzhanlong2005@126.com, ${ }^{b}$ wuliang133@189.com \\ * Corresponding Author: Liang Wu
}

\begin{abstract}
In order to solve the efficiency of buffer analysis of line elements, adopt plane graph model and according to the characteristic of simple feature model, making the boundingedges of the line elements into plane graph model, then According to certain rules to filter the lines of plane graph model to gain the bounding edges of buffer and then gain the results. In this algorithm, firstly, As required, do parallel lines and polylines vertex processing for the line elements extracted from the objects which need buffer analysis, and gain the Intermediate line objects, then Generate line elements of graph model through computing intersecting points and clip lines using static $R$ tree index, Moreover using the multiple programming standard OPENMP, Optimize the parallelism of every step of the vector buffer analysis algorithm, This algorithm can solve buffer efficient of large data and it's is better than other algorithm in physical problem.
\end{abstract}

Keywords: Simple feature model, Buffer analysis, Plane graph model, Parallel Optimization.

\section{Introduction}

Due to the importance of buffer algorithm in GIS, many scholars have done in-depth research [1-3]. Buffer analysis of vector graphics is the most basic of GIS spatial analysis function. Buffer analysis of line elements analysis is a difficult problem in vector map. In buffer analysis, often by grid method or vector method for buffer analysis of line elements, Doing radius ranging analysis, segmentation analysis of its buffer, and then get buffer radius ranging analysis. Because of lower precision grid method, it is generally by using vector methods, Vector method is the most commonly used are: Parallel double line method [4], Element superposition method [5-6], Boundary tracing method [7], However, in practical application, for varying radius buffer line features do a lot of data analysis and can not easily distinguish where the results of the analysis about the radius of buffer made, resulting in erroneous results. Therefore need to change train of thought, with the vector method of buffer analysis of line elements, Chen Youliang, research on GIS linear buffer generation algorithm, proposed the process of linear target buffer is the process of linear target coordinate point obtains its buffer point, The key algorithm is the boundary buffer generation[8].

\section{Related Concepts}

\subsection{Planar Graph and Topological Position}

Planar graph is composed of a series of plane graph elements, planar graph elements including Nodes, Edges and Directed Edges. Nodes to record the location of itself and the directed edge set, edges record the structure of compositing 
polygonal boundary coordinates, the structure or the pro and con two Directed edges combinations. Directed edges record the two directions of edges that formed by clockwise and counterclockwise coordinate of edges structure. Topological position refers to the position relationship between elements of two planar graphs with the two plane graph, in every planar graph elements recorded in its topological position relation between two planar graphs, properties of topological position is composed of three tuple <on, left, right>, on refers to the plane graph element is in what position of (on) Planar Graphs, left refers to the plane graph elements left side is what position of Planar Graphs, right refers to the plane graph elements right side is what position of Planar Graphs, The relative position of the topological values by three tuple <Interior, Boundary, Exterior> component, and it recorded the element is in planar graphs inside, outside or boundary [9]. The algorithm using these topological positions, establishment the topological rule, from the candidate edge collection found out the results polygon edges, to form the consequences of planar graphs, and ultimately formatting the intersection of polygon.

\subsection{Algorithm Data Structure}

Unequal radius line elements of the Buffer analysis requires a suitable data structure to store the line and buffer generates a result polygon, and to carry out the correct buffer analysis operation. this algorithm storage area and line point coordinates based on point coordinate storage method, line using constituted its line coordinates representation, polygon by its boundary coordinates representation, The following figure 1 shows the storage structure and the intersection of polygon. Polygon 1 coordinate string composed by $123 \mathrm{v}_{1}, \mathrm{v}_{2}, \ldots, \mathrm{v}_{8}$, the polygon based on the polygon monotonous chain segmentation algorithm divided polygon into $\mathrm{mc}_{1}, \mathrm{mc}_{2}, \ldots, \mathrm{mc}_{4}$, the monotone chain only records the pointer coordinate sequences to save storage space.

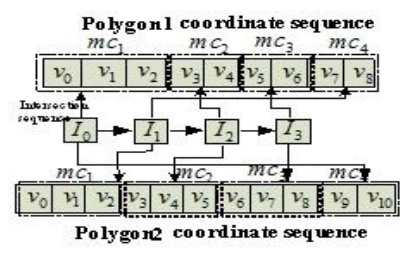

Figure 1. Polygon and Its Intersection Data Chain Diagram

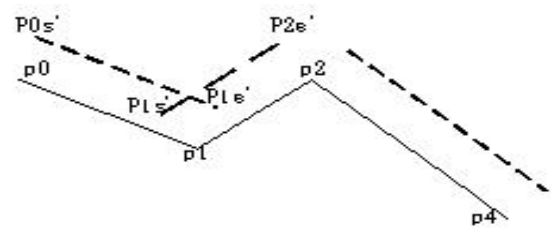

Figure 2. Buffer Sample Line Raw Data

\section{Lite Design and Implementation of Algorithms}

\subsection{Line Elements Atomic Boundary Contour Calculation}

In buffer analysis, the most common buffer styles have three kinds of cases: round, flat and square. According to whether the merger can also be divided into combined mode and do not merge mode. But in practical problems, due to the line is composed of one or a plurality of line elements, and therefore not consolidated approach is for each line buffer object to do the analysis. The combined approach is the object of all the line as a whole to buffer analysis. But in addition to its style and decide whether to merge the results of buffer analysis beyond the buffer analysis as well as the smoothness of the arc at the apex of its online elements, The following step is to calculate the boundary contour (in Figure 2 as an example). 
Calculate the left margin, lines atomic object as shown in Figure 2, a sequence of points constituting lines is $\mathrm{p} 0, \mathrm{p} 1, \mathrm{p} 2, \mathrm{p} 4$. First, make a left on the first segment of the line parallel to the line in Figure P0s'P1e ', calculation of the second paragraph of the left parallel lines $\mathrm{P} 1 \mathrm{~s}^{\prime} \mathrm{P} 2 \mathrm{e}$ ', and analyzing $\mathrm{p} 2$ relative to $\mathrm{p} 0, \mathrm{p} 1$ location, If it is located in the left, then the vertices in the vertex angle for conversion, if on the right, then to the outer angle conversion vertex, if online, then P0, P1, P2 three points collinear. When collinear, if two segments p0p1 and p1p2 only one intersection, then the two segments in the same direction, and you can think of these two segments is a segment. If there are two intersections, indicating two segments reverse, so at the apex need to add a round hat, Its second point as the starting point of a parallel line of the outer boundary contour; If it is shown in the vertex angle conversion vertices p1, then calculate the intersection of two parallel segments, the intersection of the first vertex as the outer boundary contour. If it is outside the vertex angle converter shown in $\mathrm{p} 2$, you need to add a round hat.

\subsection{The Intersection of Computing and Contour cut}

Contour intersection of computing is computing the intersection between each contour line, also includes a self-intersecting contour intersection. In seeking each segment intersection in the process, there are many possible ways to calculate, we can calculate the intersection intersect paragraph by paragraph, you can find the intersection of the scan lines by classical algorithms between each line and each line, but also can be increased by indexing demand hits two lines intersect intersection. In this paper we use the index to be calculated STR. STR is a static division of R-tree indexes. It can target line features, split on the line feature indexing process in order to reduce the intersection of intersection number. In this paper, using the method of monotonous chain constraints were strengthened to be divided on the lines. Since the R-tree is static, and therefore only considers the query, delete and add not considered, but the static R-tree can be established more quickly and query improves the efficiency for the intersection.

\subsection{Chart and Sub Graph Generation}

A plan is composed by the side, directed edges and nodes. Will be 4.2 when cut line(hereinafter called broken line) generated map. The process is as follows: Take one of the polyline called side, forward and backward with the establishment of its edge under the edge, with the same side to side to side and different symmetry called directed edges, opposite its direction. Set its relationship to the edge and the two edges, that is edges can be made according to directional edges, which can also be made symmetrical directional edge. Add a directed edge to the drawing, which is the starting point of the directed edge node, if the graph does not exist in the node, the new node will be create, and set the node of the edge of the same side of the starting point coordinates nodes. At the same time as the side edge of the node and the edge node in accordance with the right direction from the horizontal direction as a starting, and sorted according to the size of the angle of counterclockwise rotation. Figure 3 below, at the node $A$ has edges to the sequence as follows (Figure $3 \mathrm{~d} 1 . \mathrm{d} 4$ representative directed arcs, A representative node): 


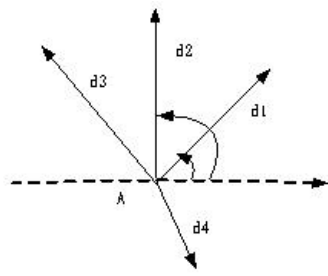

Figure 3. The Arc Sorting Example at Node

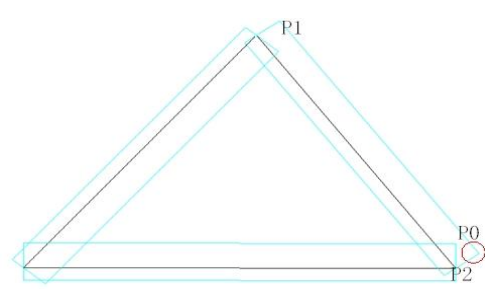

Figure 4. The Sample of Initial Determination of the Rightmost Point and Edge

It can be seen from, there can be obtained based on the directed edge corresponding to the edge and the edge nodes have to be obtained from the corresponding node based on the node point of the edge to the edge. At this point, the entire plan follow the steps above to generate complete.

Based on the plane generation sub graph, sub-graph is a subset of the planar graph, each sub-graph corresponds to a polygon, different sub graph eventually generate different polygons. The generating process of sub graph as follows: get the first node according to the node, establishing a buffer zone sub graphs, which use the container used to store the node, with a flag to record whether the node has been added, if the container inside the existence of node then sequentially take out, add the node to the sub-graph, and record the node has been added. According to the has been taken out node, obtains corresponding all directed edges, and take the directed edges of the symmetric boundary node, add it to the container, in order traversal, get all the related nodes and edges, until the container is empty now, finally got the generates a subset of planar graphs, i.e., a buffer on the map.

According to the sub-graph, the sub-graph of each with directed edges corresponding to edges with a traverse to the edge, get to the coordinates of the point on the right side, and get the corresponding to the coordinates of the points of directed edges. This need to pay attention to when the vertex is a node, i.e. the vertex index 0 , the need to judge the right most point belongs to the node where a directed edge. As shown in Figure 4, for the vertex P0, the point is that the vertices of $\mathrm{P} 1 \mathrm{P} 0$ is a directed edge vertices of $\mathrm{P} 0 \mathrm{P} 2$, but its right most side how to define? The solution here is to take the vertex P0 the corresponding node of the directed edges, take the first directed edges and finally to 0 or 1 , then the initial edge as the right most side. If the two sides of the quadrant value is 2 or 3 , then finally a directed edge is the right edge. Otherwise, take both sides according to the order of first level that not edge as the right most edge, wherein the P0P1 diagram for the right most side. At the same time the corresponding edges to vertices are different. The new index value for the last point P0P1 corresponds to the edge of the index value.

If the point is a directed edge interior point in Figure 5, the need to identify the point belongs to which section. If a before the line segment point and a point in the line above or below, need according to their relative direction to decide which is the most right point, if the point of the previous points or after a point than the rightmost point $\mathrm{Y}$ value simultaneously big or small is also need the following processing mode. If simultaneously big, you need to calculate in the right most point, constitute its next point and the previous point direction is clockwise, if, as shown in Figure 5 on the left, you need to index into the record of the previous point. Similarly, if simultaneously small, also calculate the rightmost point, whether the next point and the previous point is clockwise, if, as shown in Figure 5 on the right, the same will index move forward one. Others, still records the original dot. 
The arrows represent the direction of lines, minCoor represents the rightmost point, pNext represents the next point, pRrve represents the former one.
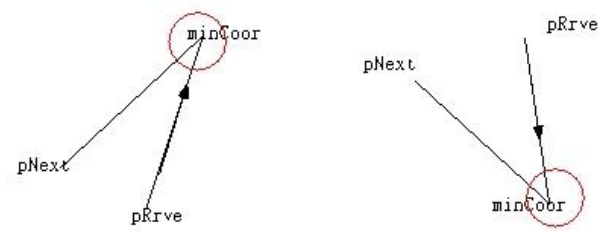

\section{Figure 5. The Sample of the Rightmost edge's Determination}

After obtaining the right most edge and the right most point, calculate the starting edge, the starting edge for the rightmost corresponds to a counterclockwise direction side. The rules are as follows: if the rightmost vertex compared with the next vertex, if the $y$ value is smaller than the next point of $Y$, then the edge that is, as the starting side, if the y value than the next point y value, then reverse the edge as the starting side, otherwise the previous point will be compared with the next point, and then obtain the starting edge.

\subsection{The Results of Calculation of Polygon Boundary}

Each sub-graph is a buffer polygon analysis results, where the operation to obtain the final one or more polygons results for each sub-diagram.

Taken out rightmost vertex which is according to Figure 4.2 in the process of establishing the sub-graph calculated. Calculating the depth values of the vertices, calculate the depth value relating its sub-graphs sorted, To calculate the depth value, we must first make the following filters for the front sub-graph (apparently already processed)same as the original side direction directed edge: If the left side of the line at that point in a certain side of the composition of successive points, and the $y$ value of the point in the $\mathrm{Y}$ value of the segment between the horizontal line is not to determine the composition of the two segments is based on the coordinates of the point whether the $\mathrm{Y}$ value strictly increasing, if the depth value is then taken to the left of the edge, if strictly decreasing, then whichever right depth value, as an alternative to the depth value, and the point as its alternative interchangeable segments, and horizontal line segments are ignored. All such segments together and depth values, select the leftmost segment depth value as the depth of its initial value rightmost. If such a line is not present, then the depth value of 0.As shown in Figure 6: the black line represents the analysis was conducted buffer line elements, the arrow represents the direction line, green lines represent the line that for the line elements conduct contour lines calculating and cutting, red virtual coil represents the line endpoints, red real circle represent the sub-graph rightmost dots, the entire map is a plane, the outside connectivity part is a child diagram, the inside connectivity part is a child diagram too, when sub-graph after sorting, the outermost sub-graph to the right of the initial value of the depth boundary is clearly 0 , and in the calculation of the second sub-graphs starting depth values, there can be seen p3 is the second sub graphs with right end point, has a solid red circle line, from $\mathrm{p} 4$ to p1 edge is its starting edge, making the calculation of depth values, it can be seen for the points $\mathrm{p} 3$, in front of it falls sort sub-graph forward directed edge only q2, q3 and q5q6 in two different directed segments of the bounding box, and left in the two segments. Since q5 and q6 are two strictly increasing, so whichever depth left as a starting depth values alternative. For $\mathrm{q} 2 \mathrm{q} 3$, because it is strictly decreasing, so whichever depth value on the right as the starting depth alternative value, and because the two points are on the left q2q3 q5q6, i.e. q5q6 alternative segment is the 
leftmost line, so its depth value as the second sub-alternative starting depth values to the right of figure.

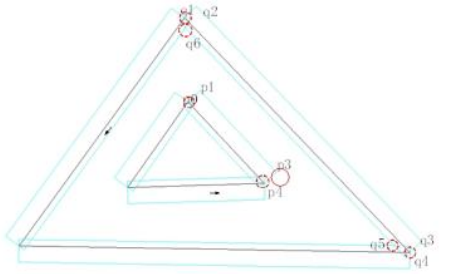

Figure 6. Depth Value Calculation Example

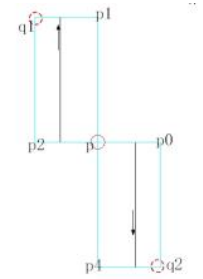

\section{Figure 7. Calculation of the Maximum Edge's Ring}

Calculating the depth of each side of the sub-graph, a directed edge is defined as the depth from the initial node of a directed edge from the directed edges, in the sub-graph of the starting node has all of the same direction to the sides there are different to the side and directed edges of the poor. Deep Computing edge in two ways, according to the directed edges with the same set of anisotropic directed edge depth, and according to the Anisotropic edge to a directed edge to set the same to a directed edge (at the same depth to the directed edges called left, anisotropic directed edges called right, its depth into the left or right depth). According to the right of the left is as follows: first, get to the directed edge of the depth factor, if it is reverse with the original edge, then the depth factor reverse, left right depth is the right depth adding depth factor, according to the difference on the left is right, right depth is left depth and depth factor difference. In calculating the depth of all the sides of the first sub-graph, calculated from the starting edge of the first, obtaining a start 4.2 establishment process of the sub-graph edge based on the calculated depth value is set to its starting depth of 0 to the right, and then set the depth of the left to the right according to depth. The symmetry of a directed edge around while the depth value with the directed depth edge is just the opposite. On the other nodes in the directed edge depth calculation also need to first calculate its already set the depth of the directed edges as the starting side, then seek the directed edges in depth.

Directed edge depth determines its location is relative to the plan view. Therefore we can get composition of the boundary contour results according to the depth value. In sub-graph, an even number of nodes by a directed edge, i.e. a number equal to the number of the incoming edge of the outside edge, because the buffer analysis retained is the outermost boundary line, which boundary line is thus left by the depth of not less than 1, the depth of which is not greater than 0 the left, and its depth is not 0 or the left to the right than the internal contour line to form, thereby obtaining a results polygon boundary.

\subsection{The Results of Polygons Calculation}

Results polygon contours 4.3 has been selected by out, then you need to have these scattered according to a certain order to the sideline to connect, and then be able to form the largest side of the ring. First taken out a node, to the node of the nodes as directed edge judgment, if the directed edge to edge or the symmetry directed edges is all the edges of the results edges, then it will be all together. For a node, there is a selvedge edge there must be an out, and the nodes are taken out of the side of the rim, so taken out the first one directed edges assumed to be out of the side edge, if that is the edge the results side, then the purpose of seeking to convert into the side, if the edge is the result of a non-edge into the side, its purpose is to 
convert it to find out the edges. A node can have multiple outgoing edges and into the side, you can also only one out of the side and into the side.

As shown in Figure 7, the black line represents the original line feature buffer analysis needs to be done, the direction of the arrow represents the direction of the line elements, (by, respectively, can be seen two lines up and down), the broken line circles represent the line break, solid red circles represent nodes. For the node $\mathrm{P}$, the point $\mathrm{P}$ to the junction edge node or a node $\mathrm{P}$ directed edge four were: $\mathrm{pp} 0(\mathrm{~T}), \mathrm{pp} 1$ $(\mathrm{F}), \mathrm{pp} 2(\mathrm{~T}), \mathrm{pp} 4(\mathrm{~F}), \mathrm{T}$ and $\mathrm{F}$ parentheses represent the original side with forward and reverse. First, the first side that pp0 (T) because it is the result of edge, so its edge as the first out edge. Since the second side pp1 (F) is not a result edge and its symmetrical directed side is the result of edge, so the symmetric directed edges as the selvedge. That is pp1 as the incoming side of the status changes to find out the side, because the third side pp2 (T) is the result of edge, so the edge pp1 next connection is set to outside edge pp2 (T). The state changed Looking selvedge for the fourth directed edge pp4 $(\mathrm{F})$, which is a result edge of the reverse edge, so it is the selvedge, the edge of the state to find out edge, because of all the nodes have been traversed to the edge so set selvedge of the next connection for the first out the side edges That is pp0 $(\mathrm{T})$. This completes the connection side of the junction point P.

This sets outside edge and the selvedge of a junction sequence, and finally set up an edge order of edge can form a closed loop. According to this order to build the largest closed boundary line, is about these side in accordance with the order of connection just turn connected to form a closed loop. As shown above, the final ring is $\mathrm{q} 1 \mathrm{p} 1 \mathrm{pp} 2 \mathrm{q} 1$ and $\mathrm{pp} 0 \mathrm{q} 2 \mathrm{p} 4 \mathrm{p}$.

After the establishment of the largest ring, you need to analyze the largest ring, if it is not 1 degree, you need to break down the ring, can be seen from the figure, after generating the largest ring, but it obviously cannot be generated based on the above link forming a result, while the need for further analysis procedure is as follows:

To take the edge the ring directed edge first, then take the directed edge the junction, and then take out the node to the edge, calculating the degree of the node of directed edge according to the degree of the node, and calculated as follows: If the directed edges of the edge ring is a ring, then the degree of the node is increased to the side ring 1 . Similarly calculated for all the other nodes of the directed edge node, will form the edge ring as the maximum degree of the edge ring. Of the edge ring may be less than 1 or greater than 1 .

As shown in Figure 8,black line shown on p10p1, p10p7, p7p4, p1p4 four line elements buffer analysis, the resulting red line two large rings, as shown in the following figure Aq1q2 ..... p12 and shown in the figure below the yellow line Bq2 ... $\mathrm{q} 1$. To calculate the degree $\mathrm{Bq} 2$... $\mathrm{q} 1$ ring as an example, the edge ring by seven edges composed, namely Bq2, q2q3, q3q4, q4Q, Qq5q6, q6q7Q, Qq1. Take the first side ring directed edge i.e. $\mathrm{Bq} 2$, then take node $\mathrm{B}$, However node $\mathrm{B}$ have four edges, wherein the edges of the ring is only $\mathrm{Bq} 2$ the side ring, so its degree is 1 , others of the same, but in the degree of computing nodes $\mathrm{Q}$, take the node of directed edges that has the edge with four Qq1, Qq7, Qq5, Qq4. Qq1 and side ring Qq5 here is that the side ring, so this degree is 2 , so this side of the ring when the maximum degree of nodes $\mathrm{Q}$ of degree 2 .

If it is greater than the degree of a node, then starting the minimum edge ring connection, establishing a minimum of side ring, according to the edge of the ring clockwise or counterclockwise to determine whether it is the case, if there is no case, then all as a hole, otherwise, the edges of the shell and the smallest ring set relations and shell holes. 
For Figure 8 degree two side ring $\mathrm{Bq} 2$. q1. First connect the smallest side ring, the steps are as follows: First, take the first side edge of the ring i.e. Bq2, then take node $\mathrm{B}$, and take out the directed edge node, the node has four directional edge, connecting the directed edge of the smallest side, taking to the second side $\mathrm{Bq} 2$ for out edge, because of its side for the side ring, so as the first one outside edges. Then look for selvedge, taking the third selvedge of q1Q, which has symmetrical edges directed edges of selvedge for the side ring and set it to the selvedge. Set status to find out the outside edges, because the fourth edge for the edges of the ring does not ring, so setting a selvedge Qq1 (F) for reverse Qq1 as Bq2, setting an edge Bq2 empathy for $\mathrm{q} 3 \mathrm{q} 4$ set $\mathrm{q} 3 \mathrm{q} 4$ edge for the next $\mathrm{q} 4 \mathrm{Q}$. In the node $\mathrm{Q}$, the directed edges were four for Qq1, Qq7, Qq5, and Qq4. Take the first directed edge Qq1, because of its the edge ring on the edge, so the side for the first outside edge, now the state is looking into the incoming side, on the second sides of Qq7, because of its not the edge ring side, while the reverse is the edge ring edge, so the edge Qq7 (F) symmetry edge, status changed to find out the outside edge, third edge Qq5 (T), due to the state change on the edge for the edge, so set into the side of the Qq7 (F) symmetry side next to the outside edge that is Qq5 (T). State to look for the edge, take the fourth side Qq4 (F), due to the symmetry of the side edges of the side of the ring's edge, so that side of things into the side of the state to find the outside side, because all the nodes has been traversing the directed edge is completed, it is finally set incoming side next to the first one outside edge for that Qq1, thus the new minimum side ring is set up, this time the two sides to form a ring, Bq2q3q4Qq1 clockwise and counter that hour of q6q7Qq5q6.

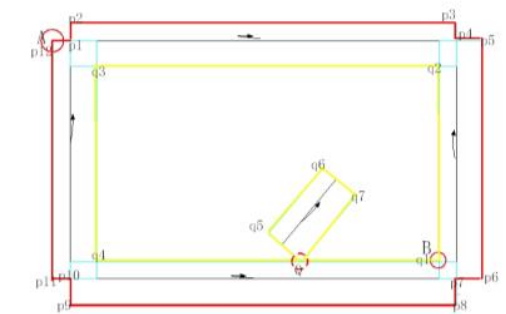

Figure 8. The Sample Data of Minimum Edge's Ring Calculation

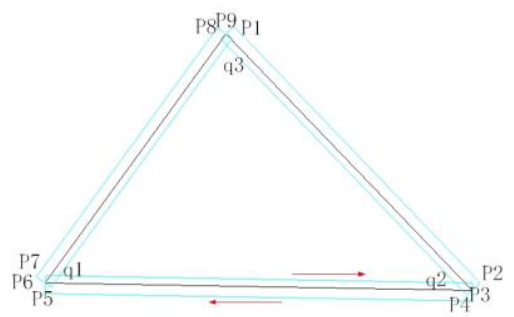

\section{Figure 9. The Holes and Shells Judgment Examples}

If the degree of node which is not greater than 1, then the largest side ring referred to as the final edge of the ring. On the edge of the ring area are classified into the shell and the hole. First, determine whether each ring composed of polygons is reasonable, that is, whether a narrow area, if it is a narrow zone, this will be ignored. Then, if it is a counter-clockwise, this is the hole, if it is smooth hour is the case, this will all be classified into the side ring and shell holes. Figure 9, there are two side ring, red arrows represent their direction, outside p1p2 ... p9p10 is (p10 and $\mathrm{p} 1$ same clockwise, Figure 9 is not shown), so as a shell, and the inside side ring q1q2q3q4, same q4 and q1, not marked in Figure 9, due to its anti-clockwise so as hole.

Then assigned to the corresponding shell hole, the hole in the shell and put away. First STR build housing index, used to quickly filter those that do not have fellowship with shell holes. Then for each hole to find the corresponding shell, as follows: for the rings hole generating a polygon, and calculating the internal point of polygon, the point extended to within the tolerance range, then bounding the point to query, access points fall into the shell, and then looking for the ultimate shell. Here there are two issues that need attention: First, calculate the polygon interior points, the second is to find the most appropriate housing, for the first 
question, first of all will be the side ring hole constitute polygon, rectangle outsourcing level achieved two sub-lines, then half of the horizontal line of the polygon and the cross-hole configuration is evaluated, the result of its calculation in which the width of the largest object, remove the object's bounding box, for the bounding box, if its width is greater than the minimum width, then the outsourcing rectangle in the middle point is an interior point of the polygon.

Level two-point line and polygon composed of the hole intersection as follows: First endpoint as the intersection of two sub-line is inserted into the drawing, then the polygon nodes as nodes into the graph; Calculation of two sub-line selfintersecting intersection and self-intersection of the intersection inserted into the diagram. For self-intersecting polygon calculating the intersection, the selfintersection of the intersection inserted into the chart; calculating the equinoctial line and polygon intersection, and inserted the intersection into diagram. Segmenting the edge of the equinoctial line, splitting the polygon edges, and then inserting these edges into the graph, where edges are not repeated edge, and then calculated the label according to depth. Remove the conflicting sides. Adding to the edges in the graph, calculate the identification point, identify incomplete node, find the results of the polygon edges cancel repeatable results edges (if the results have to side with the symmetry of the directed edge is results edge to edge which are not the result). Finally, according to the order of the first polygon, then the line and then point establish the results. Lastly, take out the center point of the outsourcing polygon, which is the results of the width of the largest results, as the polygon within-point.

Shown in Figure 10, A is a polygon with holes, B is its outsourcing rectangular equinoctial line, half line and polygon intersection get two segments q1q2 and q3q4, it can be seen from Figure $10 \mathrm{q} 1 \mathrm{q} 2$ width (i.e. outside of the rectangle $\mathrm{x}$ difference between the maximum and minimum values of $x$ ) is greater than the width of $q 3 \mathrm{q} 4$, so q1q2 is the maximum width of the target result, take bounding box that is shown by the position of the midpoint of the cross marked red circle is the within-point of the polygon.

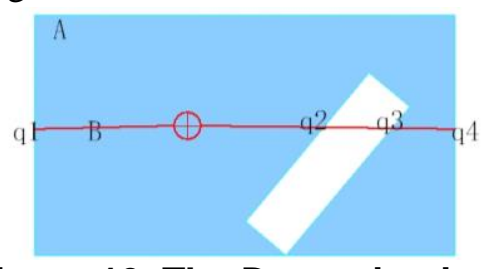

Figure 10. The Determination Example of the Identification Points

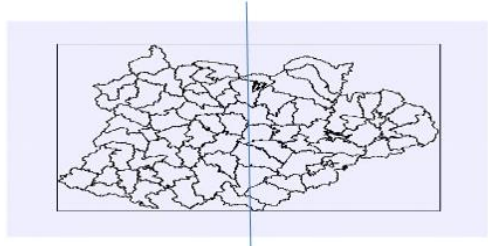

Figure 11. Space Zoning

For the second problem, the most suitable housing refers to the smallest of all shell contains the hole in the shell, the method of seeking the most appropriate shell is: with the above-obtained internal point, do the rectangular tolerance, and then use the STR rectangle by just created an index to filter part of the shell and does not cover the hole, would have covered all shells are compared to find out which one is the smallest of the shell hole.

According to the above-obtained hole and its corresponding shell, and ultimately generate a corresponding polygon, this buffer been analyzed.

\section{Parallel Optimization Analysis}

This section also uses OpenMP parallel programming specification, analysis of the previous section, ranging analysis algorithms buffer radius of each step can parallelism, looking for parallel optimization part. 


\subsection{Boundary Contour Line Features Atoms Parallel Optimization Calculation Steps}

Analysis: This part of the operation are independent, you can call multiple threads to separate atoms calculated for each element boundary contour line. This part may achieve better enhance the speed, for a line atom parallelization process, its left and right buffer radius analysis can be carried out simultaneously, such as a thread left radius buffer calculations, a thread boundary contour of the right radius buffer calculations, and finally combined multiple threads contours by the main thread. When a line for making atomic parallelism can also be a line segment (between two points that contains parts of two points) are calculated simultaneously left and right parallel lines, then its intersection and vertex processing. Finally, generating finally outer boundary of the contour at the two end points depending on how to generate a buffer.

\subsection{Contour Point Calculations and Cut Steps Parallel Optimization}

Analysis: This step is divided into two parts, and is in accordance with the first intersection point, then according to the point of intersection to cut each contour line, the cut point except outside there is no longer the intersection line. In the calculation for the intersection, and based on data parallel, i.e. the entire space region divided manner. Because aggregation of spatial objects, while the ordinary way division may not take this into account, even taking into account that, for the region of space cannot be carried out without hesitation division, also need to consider treatment at demarcation. With this in mind, we use the data at the boundary is repeated a plurality of times and as a unique identifier to distinguish the object space strategy. Shown in Figure 11 was added under the picture frame is cut after the polyline outline, while the blue vertical line in the middle of the dividing line as division data, then passes through the intermediate portion, the two nodes are calculated need to be calculated, the calculated intersection while recording a line of unique identification ID, line segment at the point of their intersection (two points for each line segment considered) position, and the first point of the line segment, and use this identification to distinguish the final the intersection of the line features.

After calculating the intersection is completed, the need for line cut operation during the cut line, the intersection point of intersection there are two possibilities, one is self-intersecting lines and the other one is the line intersects the line. But no matter what kind, intersection of a line can always sort order along the line. In the intersection sorting process, according to the intersection point of line segment record index number, and when the index number is equal to the size of the distance from the starting point to sort, after sorted, may be based on the intersection line cut. Shearing process according to the intersection of the intersection of the front and rear ends to the center in parallel proceed.

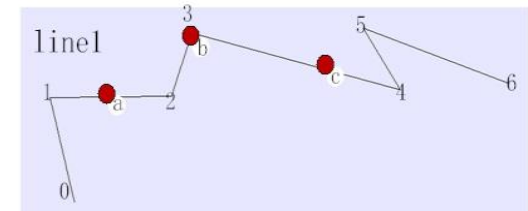

Figure 12. Sort and Cut on the Intersection's Generation Line

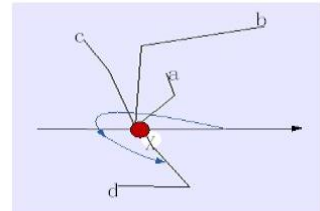

Figure 13. Sort Directed Arc at Node

In Figure 12, line 1 by line $(0,1 \ldots \ldots 6)$, seven point composition. According to the intersection of the structure can know the index intersection a of the line is 1 , 
distance from 1 point distance is denoted as $\mathrm{X}$, its front (may be equal) point is the point of 1 . The intersection points $B$ and 3 coincidence, the intersection index is 3 , the front of the point index is 3, and the point of intersection of 3 index is 3 , the front of the point of the index is 3 . To sort the three intersection points, by its index sequence can know a at the top, for b, c two intersections, the online serial numbers are equal, but due to point $b$ is smaller than the distance $c$ point distance on the 3rd point. Therefore the point $b$ at the front of the point $c$, so that the final order of the three intersection is a, b, c. By the three points can know, because no one is the intersection of two endpoints of line segments, so according to the three point of intersection, the Line 1 of the graph is divided into four segments, the first recorded as 0,1 , A. second is a, 2, B, third B, fourth $\mathrm{C}, \mathrm{C}$ from the two end, 4,5,6. can begin parallel computing, namely the a point is obtained first line, point $\mathrm{C}$ gets fourth line, followed by analogy.

\subsection{Graph and Sub-graph Generation Steps Parallel Optimization}

Graph generation, by the serial part can know, the actual process is on the whole line feature categories are generated to the arcs and nodes. In this process, the segment is a direction, here called the arcs, each line of the original are virtual into arc segment two in the opposite direction, the two arc but in a different direction, the coordinates of points are exactly the same, any arc corresponds to a line, but a line segment corresponding is the two one directed arc segment. Arc node is the start point of arc, an arc corresponds to a node, the starting point may have a plurality of arcs of the same, and therefore, a node might correspond to a plurality of arc. Because each line between is independent, so for the directed arc generation, can use the parallel way. By may know, a number of directed arc is 2 times the number of line feature class line objects. For node generation, by the above described can know, the arc starting point is the node, so for the same starting point directed arcs (that is, the node of the directed arcs), and the node of the directed arcs in accordance with the horizontal axis $\mathrm{x}$-axis positive direction as the initial direction, counterclockwise arc by size sort.

As shown in Figure 13, the starting points of four directed arcs of a, b, c and d is $\mathrm{X}$, therefore the directed arc segment of note $\mathrm{x}$ is $\mathrm{a}, \mathrm{b}, \mathrm{c}$ and $\mathrm{d}$. Also, because starting from the $\mathrm{x}$ axis positive direction, the last paragraph of four directed arcs (excluding a case where two points within a tolerance range equal to) the size of the arc so as a, b, c, d, so that at the junction $\mathrm{X}$ directed arc as a, b, c, d.

Sub-graph is a subset graph, i.e. may be a proper subset may be just a subset. In the entire graph sub-graph is a collection of all the edge points and the associated node. Shown in Figure 14, the left is the right of a sub-graph, the red represents the junction, the green line represents the contour lines, black lines with arrows represent directed arcs, and direction arrows represent the direction of the directed arcs.

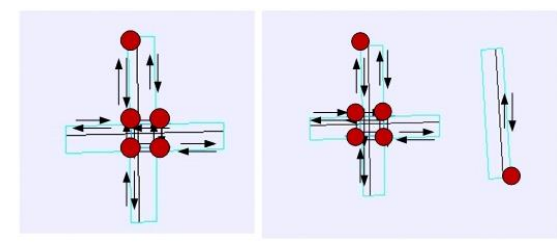

\section{Figure 14. Sub-graph Examples}

After counting the number of sub-graphs, each sub-graph is independent, it can be calculated separately rightmost vertex, and obtained the right side of the 
rightmost directed edges as outermost starting edge. For each sub-graph, we sort it according to the size value of the rightmost vertex $\mathrm{x}$.

\subsection{The Results Calculating of Polygon Boundary}

For each sub-graph, we calculated for each sub-graph corresponding to the polygon. By the serial portion may know the calculation of a sub-graph, first need to calculate the depth value of its initial edge, since it depends on the sequence subgraph, so the sub-graph polygons calculated cannot be parallel. However, in the same sub-figure, which is implied good parallelism.

First, after obtaining a start edge depth value, since a depth value to the directed edges can be obtained based on their symmetry, that mutually symmetrical directed edge also symmetrical to each other left and right depth values. In the node directed arc segment, if the start point of the arc is the point, it is considered that the directed arcs of the node are the point, and the node is included in the directed arcs of the directed arc. Also, because the size of the directed arcs node is a starting side of the $\mathrm{x}$-axis positive direction is calculated in accordance with the reverse curvature of the sort, For a node, the next of the first node directed arc is the second, the next arc of the last arcs is first, that is, for a node, its relationship between the directed arcs are equal, there is no primary and secondary, no order, just for the convenience of calculation them according to certain rules of the sort, and may be considered to be a circular sequence, each directed arc of the node (with the number of arcs is more than one) has the next directional arc, as shown in Figure 15. It can know, find the value of the junction depth of the directed arc, the depth value from any one of directed arcs can launch depth values of the curvature of the other node. In a graph, know depth the starting side, it means to know the depth value of the two directed arcs, that is, at the same time be able to calculate the depth value directed arcs two nodes, and so on, can all junction depth value is calculated, while using a record which identifies a node to have been calculated to avoid duplication of calculations. According to its depth value, the result will be the directed arc edge saved.
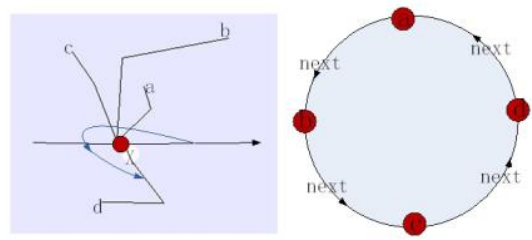

Figure 15. Calculation of the Radian Value

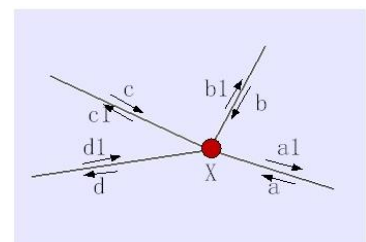

Figure 16. Edge Sort

\subsection{Parallel Optimization Step Results Polygon}

In the figure, the performance of a polygon ring, for a directed graph, the polygon can be expressed in the same direction as a series of directed edges in the polygon, any of a polygon vertex is connected to at least two segments, Thus the composition of the two endpoints of the polygon edges are no exception, they are connected to at least two sides, in the directed graph can be represented by polygons in the same direction as a series of directed edges known, any one of the polygon are that points to the ends of the arc node has at least two have connected to the arcs, that at least one of the edges and one outgoing edge.

As shown in Figure 16, node X at four directed arcs, respectively, a1, b1, c1, d1. Its first directed edge (an outgoing edge) is b1, find out into the side is a, next there is a directed edge $\mathrm{c} 1$, which is the side $\mathrm{b}$, a third directed edge is $\mathrm{d}$, which is the side 
c, the last one is directed edge a1, which is the side $d 1$. This establishes the $X$ over the edge node sequence is (assuming from a start): A-> b1, b-> c1, c-> d, d1-> a1 according to the order of edges between nodes, can form one or more closed rings, between the respective nodes is independent, and therefore can calculate the degree of each node, if it is greater than the one it is decomposed otherwise the polygon constituting the ring is a ring. Then, for each ring to judge, by a clockwise or counterclockwise direction will be divided into a polygonal or polygon housing hole, and then allocated to the respective hole in the corresponding shell, and finally to form the final polygon from shells.

\section{Algorithm Analysis And Test Results}

Experiments on multi-core platforms Windows XP system, using C / C ++ language, this paper using MapGIS platform, according to algorithm design and analysis of parallel optimization realize it and conducted a experiment. The experiment was conducted on a dual-core processor, these experiment configurations of the machine are: processor model, the memory for $2 \mathrm{G}, 500 \mathrm{G}$ hard drive for software: The operating system is Windows XP Gohost 1.0, the compiler environment vs 2005 .

This section focuses on description of the parallel test radius buffers ranging in multicore processors ranging from the environment than the serial radius buffer analysis more efficient.

Because the Buffer radius ranging not only is data-intensive algorithm also is computationally intensive algorithm, so this section were tested by 77 (self-made data), 239 (self-made data), 724 (GDB: // Wuhan / city centerline), 1084 (self-made data) of the amount of data results are shown below:

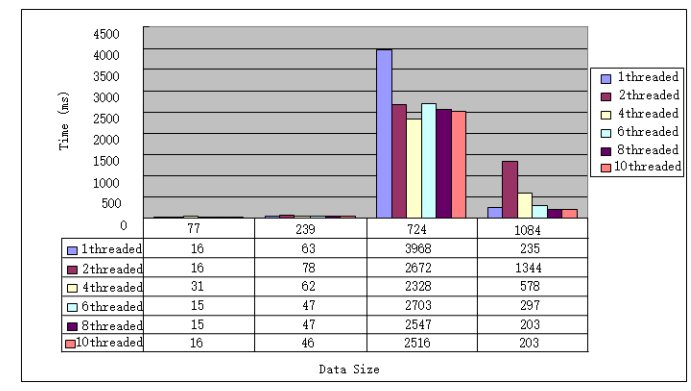

\section{Figure 17. The Buffer Analysis of Different Threads on Quad-core Processors}

Not only the size of the data lines and the number of relationships, and a relationship with the points of composition lines, the more lines of points, the greater the amount of data, the amount of data for the data in this experiment 724 actually lines up the number of points the data, therefore, become the most time is data used, but here for convenience, not for each line the number of statistical points, and therefore only the number of lines to represent the size of the data. As can be seen from Figure 17, the data than the smaller 77-core 239, due to exceptionally low serial core parallel algorithms with time, and therefore did not see any advantage, but in 1084 when the amount of data is due to a single thread has a very high efficiency, whereas in the case of multi-threaded but because the communication between threads so that the efficiency but lower or no obvious advantage, but the amount of data line 724 , since it points more, but the amount of data comparison large, so the parallel to serial exhibit performance advantages. 
Figure can be obtained by the different amount of data corresponding to the speedup. Shown as table 1 below:

Table 1. The Serial Algorithm of Unequal Radius Buffer Analysis

\begin{tabular}{cccccc}
\hline Data Size & 2 nuclear speedup & $\begin{array}{c}4 \text { nuclear } \\
\text { speedup }\end{array}$ & $\begin{array}{c}6 \text { nuclear } \\
\text { speedup }\end{array}$ & $\begin{array}{c}\text { 8 nuclear } \\
\text { speedup }\end{array}$ & $\begin{array}{c}\text { 10 nuclear } \\
\text { speedup }\end{array}$ \\
\hline 724 & 1.485029 & 1.704467 & 1.467999 & 1.557911 & 1.577107 \\
\hline 77 & 1 & 0.516129 & 1.066667 & 1.066667 & 1 \\
\hline 239 & 0.807692 & 1.016129 & 1.340426 & 1.340426 & 1.369565 \\
\hline 1084 & 0.174851 & 0.406574 & 0.791246 & 1.157635 & 1.157635 \\
\hline
\end{tabular}

As can be seen above, the buffer varying radius ranging analysis of parallel multicore processors than the serial buffer algorithm has obvious efficiency advantage, which in the case of large amount of data. The amount of data is 724 , the two-nuclear speedup is 1.485029 , and 4-core speedup can reach 1.704467 .

As can be seen from the table, there is a very obvious speedup fluctuations, the reason may be related to the amount of data and zoning cases, if the amount of data is small, its segmentation and communication between threads occupied after making the parallel acceleration time than did get promoted, if the majority of the region need to repeat the calculation, then the computation time will not decrease, but also takes up more space. By contrast, if a large amount of data, and the division of the comparison segmentation appropriate elements that double counting is not a lot, so efficiency is relatively high.

Compared with classical algorithm, when the present algorithm for the analysis of elements need to merge buffer, do not need to merge polygon operation, and for the buffer unequal radii analysis did not requires divided the analysis of the results buffer, thus avoiding the polygon segmentation errors. This algorithm can be computed in the model in FIG edge depth values, according to the depth value of the edge to determine the outcome of the contour, then the results of the contour line processing, it produces a result of polygons. Because this algorithm using vector method, therefore the calculation is precision and high efficiency in practical applications.

\section{Summary}

This article buffer analysis algorithm is applicable to a variety of special lines. The algorithm only needs to judge the generation of the intersection exit point and entry points, simply put all the boundary points of each of two polygons read out and calculated, and formatting the result polygon, Find the results polygon from the generation of candidate edges and generate results polygons, so the algorithm than the same algorithm is simple. Finally, based on the MapGIS platform, experimental the algorithm, experimental results show that the algorithm accuracy and correctness significantly better than other similar algorithms. Since there is no topological relations between the various polygon simple feature class model, so the model is easier to implement than the topological model analysis buffer operator parallelization operation. In multicore processors, parallel buffer radius ranging analysis has been greatly speedup and improves the performance of spatial analysis. This should be attributed parallelism and spatial data, make parallel unequal radius buffer analysis accelerate better, performance than the radius ranging from serial buffer analysis. 


\section{Acknowledgement}

This research work supported by Research Programs national natural science foundation of China (41401443) and Fundamental Research Funds for the Geographic Information Engineering and National Key Laboratory Fund (No.SKLGIE2013-Z-4-1) and Information Engineering in Surveying, State Key Laboratory funded projects (No 13I02) and Research Funds for the Central Universities basic special projects No. CUG160226.

\section{References}

[1] H.Y. Wu and J.-Y. Gong, "Buffer Curve and Buffer Generation Algorithm in Aid of EdgeConstrained Triangle Network", Acta Geodaetica et Cartographica Sinica, vol. 28, (1999), pp. 355-359.

[2] B. Zalik and M. Zadravec, "Construction of Non-symmetric Geometric Buffer from a Ser of Line Segments", Computers \&Geo-sciences, vol. 29, (2003), pp. 53-63.

[3] W. Xiang and W. L. Stratton, "The B-Function and Variable Stream Buffer Mapping: Anoteon, A GIS Method for Riparian Water Quality Buffer Generation”, UGIS, vol. 10, no. 4, (1996), pp. 499-510.

[4] H.-H. Wu, "Problems on the establishment of GIS buffer", Geomatics and Information Science of Wuhan University, vol. 22, no. 4, (1997), pp. 358-366.

[5] H.-Y. Wu, J.-Y. Gong and D.-R. Li, "Buffer Curve and Buffer Generation Algorithm in Aid of Edge-Constrained Triangle Network", Acta Geodaetica et Cartographica Sinica, vol. 28, no. 4, (1999), pp. 355-359.

[6] H. Zhu, T.-H. Jhai and H. Wang, "The Buffer Construction of Line Object Based on the Geometric Scan Idea", Acta Geodaetica et Cartographica Sinica, vol. 22, no. 6, (2006), pp. 18$21,59$.

[7] J.-C. Wang, Y.-K. Rui and Y.-Q. Li, "Novel method for buffer gener ation based on vector boundary tracing", Computer Engineering and Applications, vol. 43, no. 33, (2007), pp. 66- 68.

[8] Y. L. Chen and H. X. Weng, "The Algorithms of Generating buffers for Linear Objecst in GIS", Journal of Jiangxi University of Science and Technology, vol. 10, no. 29, (2008), pp. 37-40.

[9] Z.-L. Chen, X.-C. Wu and L. Wu, "Polygon Overlay Analysis Algorithm Based on Monotone Chain and STR Tree in theSimple Feature Mode", Acta Geodaetica et Cartographica Sinica, vol. 1, (2010), pp. 102-108. 
International Journal of Grid and Distributed Computing Vol. 9, No. 12 (2016) 